\title{
Secondary findings from next-generation sequencing: what does actionable in childhood really mean?
}

\author{
Julie Richer, $\mathrm{MD}^{1}$ and Anne-Marie Laberge, $\mathrm{MD}, \mathrm{PhD}^{2,3}$
}

Purpose: We aimed to assess the definition of actionability of secondary findings in childhood, using a screening framework.

Methods: For 31 disorders on the American College of Medical Genetics and Genomics SF v.2.0 list, World Health Organization screening criteria were applied to assess actionability in childhood.

Results: The age of onset was variable. We categorized disorders based on the proportion of cases that presented in childhood: rare $(n=$ $6)$, fewer than half the cases $(n=9)$, the majority of cases $(n=12)$, or unclear $(n=4)$. The age at initiation of intervention was based on the youngest age of onset reported, not evidence of the benefit of early intervention. For 15 disorders, guidelines were supported by a moderate quality of evidence for at least one recommendation. Only tuberous sclerosis complex had recommendations based on high-quality evidence. All others were based on evidence of low or very low quality.
Conclusion: We propose that actionability in childhood should be based on the proportion of cases that manifest in childhood and the quality of the evidence supporting intervention recommendations. Ideally, disclosure in childhood would be limited to disorders for which a majority of cases present in childhood and for which interventions are supported by evidence of at least moderate quality (i.e., multiple endocrine neoplasia type 2, retinoblastoma, tuberous sclerosis complex, Marfan syndrome, and Wilson's disease).

Genetics in Medicine (2019) 21:124-132; https://doi.org/10.1038/s41436018-0034-4

Key words: ACMG gene list; actionability; children and adolescents; secondary findings; opportunistic screening

\section{INTRODUCTION}

Genetic diagnostic testing has progressed at an exponential pace since the advent of next-generation sequencing. ${ }^{1}$ Patients whose clinical symptoms do not suggest a recognizable syndrome can now undergo testing using increasingly more comprehensive gene panels, exome sequencing, or genome sequencing. However, such untargeted testing comes at a cost: as more genes are tested, the likelihood of identifying a potentially clinically relevant finding not related to the primary indication for testing increases. These clinically relevant secondary findings are estimated to occur in approximately $1-2 \%$ of individuals undergoing exome sequencing. ${ }^{2,3}$ The question of which secondary findings to return to patients has generated much discussion and led to formal position statements in Canada, the United States, and Europe. ${ }^{4-7}$

In 2013, the American College of Medical Genetics and Genomics (ACMG) recommended reporting-regardless of age-secondary findings that are known pathogenic or expected to be pathogenic when found in one of 57 genes associated with disorders/groups of disorders considered actionable. ${ }^{4}$ The rationale was that pathogenic variants in these genes were associated with potentially preventable health outcomes, and therefore should be reported in the best interests of the patient.

These recommendations raised dissenting voices. ${ }^{8,9}$ Concerns included whether the approach used by the ACMG represented opportunistic screening, the level of evidence available about penetrance of pathogenic variants in an unselected population, the lack of an option for patients to opt out of receiving these secondary findings, and the recommendation to disclose variants in these genes irrespective of patient age despite the fact that some of these genes lead to adult-onset disorders. Following this debate, the ACMG issued clarifications, mainly adding the right of the patient or family to opt out of the report of secondary findings. ${ }^{10}$ In 2016, they revised the list to add four genes (ATP7B, OTC, SMAD4, and BMPR1A) for three disorders (Wilson's disease, ornithine transcarbamylase deficiency, and Juvenile polyposis) and remove one gene (MYLK, associated with familial thoracic aortic aneurysms and dissections). ${ }^{7}$ The recommendation to specifically search for disease-causing and likely disease-causing pathogenic variants in the genes listed in patients undergoing exome and genome sequencing regardless of patient age was maintained.

\footnotetext{
${ }^{1}$ Department of Medical Genetics, Children's Hospital of Eastern Ontario, Ottawa, Ontario, Canada; ${ }^{2}$ Department of Pediatrics, Université de Montréal, Montréal, Quebec, Canada; ${ }^{3}$ Medical Genetics Division and Research Center, Centre Hospitalier Universitaire Sainte-Justine, Montréal, Quebec, Canada. Correspondence: A-M. Laberge (anne-marie.laberge.hsj@ssss.gouv.qc.ca)
} 
When it comes to reporting such findings in children, European recommendations are more open-ended, suggesting that "guidelines need to be established as to what unsolicited information should be disclosed to balance the autonomy and interests of the child and the parental rights and needs (not) to receive information that may be in the interest of their (future) family". ${ }^{5,11}$ Taking into consideration the Canadian health-care context, the Canadian College of Medical Geneticists took a more restrained approach by recommending disclosure of secondary findings in children in the case of highly penetrant disorders that are medically actionable during childhood. ${ }^{6}$

Traditionally, predictive testing in children has been limited to disorders that are actionable in childhood. ${ }^{12-14}$ One concern about predictive testing was the risk of genetic discrimination, especially if there are no (or limited) interventions available to improve health outcomes. In the United States, the Genetic Information Nondiscrimination Act prevents genetic discrimination in health insurance and employment decisions, but not in other settings. ${ }^{15}$ In Canada, access to health insurance is mandated by the Universal Health Care Act, and bill S201 was recently passed to protect from genetic discrimination in other settings. ${ }^{16,17}$ Nevertheless, the potential harms of disclosing to children secondary findings that will not be actionable until adulthood may include genetic discrimination for insurance and future employment, as well as potential psychosocial harms. ${ }^{18}$ It is therefore imperative to understand the meaning of "actionable in childhood" to ensure that potential harms are minimized.

An actionable finding can be defined as a disease-causing pathogenic variants for a disorder for which surveillance and preventive and/or treatment measures are available to significantly improve health outcomes associated with the disorder. ${ }^{4}$ However, this definition is subject to interpretation, in terms of what constitutes a significant impact on health outcomes. Efforts have been made to develop a more objective measure; for example, a semiquantitative metric assessing actionability based on five characteristics: severity and likelihood of the disease outcome, efficacy and burden of intervention, and knowledge base. ${ }^{19}$ Others have developed a categorization of actionability to distinguish medical actionability from patient-initiated actionability and life-plan decisions. ${ }^{20}$ In the pediatric context, additional issues complicate the application of this definition of actionability: age of onset of disease, age of onset of preventable manifestations of the disease, age at which "action" (treatment or surveillance) should be initiated, and how variable these ages can be for a given disorder or a given family.

In this context, we decided to assess the actionability of genetic disorders during childhood using a population screening perspective and the disorders included in the ACMG list as case examples. We propose (1) an approach to assessing "actionability during childhood" for disorders for which genomic testing is performed and (2) a threshold for disclosure in childhood.

\section{MATERIALS AND METHODS}

We applied a public health population-based screening perspective, using the World Health Organization screening criteria, to determine whether a disorder was a good target for opportunistic screening in the context of genome sequencing. ${ }^{21,22}$ Among the World Health Organization screening criteria, we selected criteria relating to actionability of screened disorders in childhood. For each criterion, we identified variables to assess in the disorders to be reviewed (Table 1).

We applied this framework to the 31 disorders (associated with 59 genes) considered actionable by the ACMG in the context of genomic sequencing. ${ }^{4,7}$

We reviewed the characteristics of the 31 disorders, having decided to focus on disorders, rather than genes, because actionability is based on the impact of an intervention on health outcomes, and therefore the disease process. If a disease is caused by multiple genes, but the intervention is the same for all cases of the disease, the impact of treatment/ surveillance is based on disease outcomes, not on the underlying genetic etiologies. For each disorder, we searched for published management and/or health-care supervision guidelines through the National Guideline Clearinghouse and PubMed. Guidelines that did not address surveillance or preventive management were not considered (e.g., guidelines on indications for genetic testing). For each disorder, we reviewed the most recent GeneReviews article when available. If a GeneReviews article was not available for that disorder, we searched for recent publications about the natural history and management of that disorder through PubMed.

\section{RESULTS}

Disease characteristics were summarized as they relate to childhood onset and actionability in Supplementary Tables S1 and S2, respectively.

The average age at diagnosis and penetrance of the disease (in particular age-related penetrance) help to assess the likelihood of disease occurrence in childhood. Other factors taken into account include whether the disorder is known to have inter- or intrafamilial variability, evidence of genotype-phenotype correlations for particular pathogenic variants, and the proportion of cases of the disorder that occur de novo. For a highly penetrant autosomal dominant disorder for which most cases are inherited, the likelihood of an secondary finding is low.

Based on these characteristics, we categorized the 31 disorders into 4 groups based on the likelihood of childhood onset: rare $(n=6)$, fewer than half the cases $(n=9)$, the majority of cases $(n=11)$, and unclear $(n=4)$ (Table 2). Diseases were considered "rare" in childhood if the available evidence described its onset in childhood only qualitatively as "rare" or "exceptional", because childhood onset is too uncommon to allow for a quantitative estimate of the proportion of cases that present in childhood. Even in disorders for which the majority of cases have their onset in childhood, inter/intrafamilial variability and incomplete 
Table 1 World Health Organization screening criteria related to actionability of screened disorders used to assess actionability in childhood

Characteristics of disorder related to actionability in childhood

Criteria

Related to childhood onset of disease Related to surveillance and/or treatment

(Supplementary Table S1)

(Supplementary Table S2)

\section{Classic Wilson and Jungner criteria ${ }^{22}$ \\ 1. The condition sought should be an important health problem \\ 2. There should be an accepted treatment for NA patients with recognized disease \\ 3. Facilities for diagnosis and treatment should NA be available \\ 4. There should be a recognizable latent or NA early symptomatic stage \\ 5. There should be a suitable test or examination \\ 6. The test should be acceptable to the population \\ 7. The natural history of the condition, including development from latent to declared disease, should be adequately understood}

8. There should be an agreed policy on whom to treat as patients

NA

NA

A

NA

NA

Average age at diagnosis; age-related

penetrance; $\%$ presenting before age 18;\% cases due to de novo pathogenic variants Genotype-phenotype correlation; inter/ intrafamilial variability NA

NA (s)
Availability and acceptability of surveillance and/or treatment; risks of prevention/treatment outweighed by benefits

Available surveillance and treatment

Recognizable latent or early stage; surveillance and/or treatment effective in latent or early stage

Potentially preventable/treatable health outcome

Clear treatment guidelines; quality of evidence available about effectiveness of intervention; surveillance/treatment to be initiated in childhood NA

9. The cost of case finding (including diagnosis and NA treatment of patients diagnosed) should be

economically balanced in relation to possible expenditure on medical care as a whole

10. Case finding should be a continuing process and NA

not a "once and for all" project

Emerging World Health Organization screening criteria ${ }^{23}$

The screening programme should respond to a NA recognized need

The objectives of screening should be defined at the NA outset

\begin{tabular}{|c|c|}
\hline There should be a defined target population & $\begin{array}{l}\text { Target population based on age? Other } \\
\text { indication for testing? }\end{array}$ \\
\hline $\begin{array}{l}\text { There should be scientific evidence of screening } \\
\text { programme effectiveness }\end{array}$ & NA \\
\hline
\end{tabular}

The programme should integrate education, testing, NA clinical services, and programme management

There should be quality assurance, with mechanisms NA

NA NA

to minimize potential risks of screening

The programme should ensure informed choice, NA confidentiality, and respect for autonomy

The programme should promote equity and NA access to screening for the entire target population

Programme evaluation should be planned from the NA

outset

The overall of screening should outweigh the NA harm

NA Not applicable
Potentially preventable/treatable health outcome

NA

NA

Quality of evidence available about effectiveness of intervention; surveillance and/or treatment effective in latent or early stage

NA

Impact of prevention/treatment on health and psychosocial outcomes

Acceptability of surveillance/treatment

NA

Risks of prevention/treatment outweighed by benefits 
Table 2 Disorders on the ACMG SF v2.0 list categorized based on proportion of cases with onset in childhood

\begin{tabular}{|c|c|}
\hline Onset in childhood & Disorders \\
\hline $\begin{array}{l}\text { Rare to exceptional } \\
(n=6)^{\mathrm{a}}\end{array}$ & $\begin{array}{l}\text { Hereditary breast and ovarian cancer;Lynch } \\
\text { syndrome;MUTYH-associated polyposis;PTEN } \\
\text { hamartoma tumor syndrome (rare for } \\
\text { preventable tumors); familial thoracic aortic } \\
\text { aneurysms and dissections; dilated } \\
\text { cardiomyopathy }\end{array}$ \\
\hline $\begin{array}{l}\text { Fewer than half the } \\
\text { cases }(n=9)\end{array}$ & $\begin{array}{l}\text { Li-Fraumeni syndrome; multiple endocrine } \\
\text { neoplasia type 1; hereditary paraganglioma- } \\
\text { pheochromocytoma syndrome; } \\
\text { neurofibromatosis type 2;Ehlers-Danlos } \\
\text { syndrome (vascular type); hypertrophic } \\
\text { cardiomyopathy; arrhythmogenic right } \\
\text { ventricular dysplasia;Romano-Ward long QT } \\
\text { syndrome types 1, 2, and 3;Brugada syndrome }\end{array}$ \\
\hline $\begin{array}{l}\text { Majority of cases ( } n \\
=12 \text { ) }\end{array}$ & $\begin{array}{l}\text { Familial hypercholesterolemia (complications rare } \\
\text { before adulthood);Peutz-Jeghers syndrome; } \\
\text { familial adenomatous polyposis; juvenile } \\
\text { polyposis (for polyps, but cancer rare in } \\
\text { childhood); multiple endocrine neoplasia type 2; } \\
\text { retinoblastoma; tuberous sclerosis complex;WT1- } \\
\text { related Wilms tumor;Marfan syndrome; } \\
\text { catecholaminergic polymorphic ventricular } \\
\text { tachycardia;Wilson's disease;ornithine } \\
\text { transcarbamylase deficiency (males only) }\end{array}$ \\
\hline Unclear $(n=4)$ & $\begin{array}{l}\text { Malignant hyperthermia susceptibility; Von } \\
\text { Hippel-Lindau syndrome; familial medullary } \\
\text { thyroid cancer;Loeys-Dietz syndrome }\end{array}$ \\
\hline
\end{tabular}

aDiseases were considered "rare" in childhood if the available references described its onset in childhood only qualitatively as "rare" or "exceptional", because childhood onset is too uncommon to allow for an estimation of the proportion of cases that present in childhood

penetrance can make it difficult to predict clinical outcome in a given patient.

Supplementary Table S2 summarizes what is known about prevention, surveillance, and treatment for each disorder. All disorders have potentially preventable or treatable manifestations, and surveillance and treatment is available for all disorders. However, consensus guidelines from professional societies or evidence-based guidelines about surveillance and/ or treatment are not available for all disorders. When available, some surveillance and/or treatment recommendations are based on expert opinion only. Evidence of improved health outcomes thanks to recommended surveillance and/or treatment is only available for about two-thirds of the disorders. Furthermore, even when guidelines are available, recommended surveillance or treatment strategies are not always acceptable to all, especially if they include invasive procedures. For example, elective colectomy as a preventive measure in familial adenomatous polyposis is associated with significant morbidity: it is delayed in some patients after considering the number and size of polyps and their histology.
Nevertheless, the benefits of surveillance or treatment strategies are considered to outweigh the risks in almost all disorders.

We found that the age at which initiation of surveillance and/or treatment was recommended is during childhood for almost all disorders. The exceptions are hereditary breast and ovarian cancer, Lynch syndrome, and MUTYH-associated polyposis (Supplementary Table S2). For most disorders, this seems to be based on the youngest age at which the manifestation or complication was reported in the literature, even if it is exceptional for cases to manifest in childhood. For a few disorders for which evidence is limited, the age at which surveillance or treatment should be initiated is not clearly defined, but is still suggested to begin in childhood. Whether early surveillance and intervention starting in childhood is actually effective is not clearly established for most disorders. For example, the US Preventive Services Task Force has not recommended screening for familial hypercholesterolemia because it found inadequate evidence on the association between changes in intermediate lipid outcomes or noninvasive measures of atherosclerosis in children and adolescents and the incidence of or mortality from relevant adult health outcomes. ${ }^{23}$

Table 3 summarizes the quality of evidence supporting recommendations for surveillance, prevention, and/or treatment for each disorder. For 15 disorders, guidelines are supported by a moderate quality of evidence for at least one recommendation. Only tuberous sclerosis complex has recommendations based on high-quality evidence. All others are based on evidence of low or very low quality.

\section{DISCUSSION}

We propose an approach to assessing "actionability during childhood" for disorders for which genetic or genomic testing is performed, to inform the choice of a threshold for disclosure in childhood (Table 4). As discussed in the introduction, an actionable disorder is a disorder for which surveillance and preventive and/or treatment measures are available to significantly improve health outcomes associated with the disorder. For the purpose of our analysis, we limited our assessment to medical actionability, since the disclosure of secondary findings in childhood will not impact patientinitiated actionability or life plans in the short term and is most likely to provide benefit in terms of medical actionability. ${ }^{20}$ Therefore, we consider a disorder "actionable in childhood" if there are available preventive and/or treatment measures and the disorder has either (i) childhood onset and such measures are therefore initiated in childhood, or (ii) adult onset, but such measures have been demonstrated to be effective when started in childhood. For the sake of conciseness, we use the term "preventive and/or treatment measures" to include all actions that can be taken to improve health outcomes, including prevention, surveillance, and treatment.

We used the disorders on the ACMG list as case examples to assess the actionability in childhood of genetic disorders, 
Table 3 Level of quality of evidence for each disorder, by categories of proportion of cases with onset in childhood

\begin{tabular}{|c|c|c|c|c|c|}
\hline Onset in childhood & Disorder & Very low & Low & Moderate & High \\
\hline \multirow{4}{*}{ Rare to exceptional $(n=6)$} & Lynch syndrome & $\checkmark$ & $\checkmark$ & $\checkmark$ & \\
\hline & PTEN hamartoma tumor syndrome & $\checkmark$ & & & \\
\hline & Familial thoracic aortic aneurysms and dissections & & $\checkmark$ & $\checkmark$ & \\
\hline & Dilated cardiomyopathy & & $\checkmark$ & $\checkmark$ & \\
\hline \multirow{5}{*}{ Fewer than half the cases $(n=9)$} & Multiple endocrine neoplasia type 1 & & $\checkmark$ & & \\
\hline & Hereditary paraganglioma-pheochromocytoma syndrome & $\checkmark$ & & & \\
\hline & Neurofibromatosis type 2 & $\checkmark$ & & & \\
\hline & Ehlers-Danlos syndrome (vascular type) & $\checkmark$ & & & \\
\hline & Hypertrophic cardiomyopathy & & $\checkmark$ & $\checkmark$ & \\
\hline \multirow[t]{11}{*}{ Majority of cases $(n=12)$} & Familial hypercholesterolemia & & $\checkmark$ & & \\
\hline & Peutz-Jeghers syndrome & & $\checkmark$ & & \\
\hline & Familial adenomatous polyposis & $\checkmark$ & $\checkmark$ & & \\
\hline & Juvenile polyposis & $\checkmark$ & $\checkmark$ & & \\
\hline & Multiple endocrine neoplasia type 2 & $\checkmark$ & $\checkmark$ & $\checkmark$ & \\
\hline & Retinoblastoma & & & $\checkmark$ & \\
\hline & Tuberous sclerosis complex & $\checkmark$ & $\checkmark$ & $\checkmark$ & $\checkmark$ \\
\hline & WT1-related Wilms tumor & $\checkmark$ & & & \\
\hline & Marfan syndrome & & $\checkmark$ & $\checkmark$ & \\
\hline & Catecholaminergic polymorphic ventricular tachycardia & & $\checkmark$ & & \\
\hline & Wilson's disease & & $\checkmark$ & $\checkmark$ & \\
\hline
\end{tabular}

through a population screening criteria perspective. Our analysis is limited to actionability in childhood, which implies that we have purposefully not considered factors that would need to be considered if assessing the relevance of population screening for these disorders in general. For example, based on Wilson and Jungner screening criteria, the cost of case finding using such technology would need to be balanced with expenditures on medical care as a whole. Also, in line with current recommendations about predictive genetic testing in children, we focused on the benefits of actionability for the child, not for the family. ${ }^{12-14,24}$ According to the ACMG, some of the 31 genes were included because the disclosure of a pathogenic variation in the child may benefit the parent and thereby the child by preserving the family unit. While we are not disputing that in some circumstances such an indirect benefit may be in the best interests of the child, we limited our assessment here to actionability for the child.

As shown, childhood-onset cases have been reported for the majority of the disorders included in the ACMG gene list (Supplementary Table S2). However, we argue that the proportion of cases that would benefit from an intervention in childhood varies greatly from one disorder to another, depending on the proportion of cases that typically present in childhood (Table 2 and Supplementary Table S1). For example, pathogenic variants in $R B 1$ (the gene associated with retinoblastoma) are clearly actionable in childhood, as practically all cases present in early childhood. In comparison, the penetrance of multiple endocrine neoplasia type 1 is $50 \%$ by age 20 , and hereditary breast and ovarian cancer is exceptionally rare before age 18 . Another issue is the difference between the age of onset of disease and the age at presentation of preventable complications. For example, familial hypercholesterolemia can be diagnosed as early as 2 years of age, but complications in the form of coronary heart disease are seen in about $50 \%$ of males by age 50 . Treatment with statins is currently not recommended before eight years of age. In familial adenomatous polyposis, the age of onset of polyps is in childhood; surveillance is recommended from ten years of age, but cancer most often presents in adulthood. When is the disease 
Table 4 Assessment of actionability in childhood

Definition of actionability in childhood
(i) Preventive and/or treatment measures are available to significantly
improve health outcomes associated with the condition
$\begin{aligned} & \text { (ii) The condition has childhood onset and such measures are therefore } \\ & \text { initiated in childhood, or the condition has adult onset, but preventive } \\ & \text { measures are proven effective when started in childhood }\end{aligned}$
$\begin{aligned} & \text { Questions to ask when assessing a condition's actionability in } \\ & \text { childhood }\end{aligned}$
$\begin{array}{ll}\text { Age of onset } & \text { What is the age of onset? } \\ \text { Is it uniformly in childhood? } & \text { Or is there only a subset of cases that present } \\ \text { in childhood? } \\ \text { What is the proportion of cases that present in } \\ \text { childhood? } \\ \text { Is the proportion of cases with onset in } \\ \text { childhood high enough to justify initiating } \\ \text { prevention and/or surveillance in an } \\ \text { asymptomatic child? }\end{array}$
$\begin{aligned} & \text { What are the preventive and/or treatment } \\ & \text { measures available in childhood? } \\ & \text { How effective are they in children? } \\ & \text { What is the quality of the evidence to support } \\ & \text { their effectiveness in childhood? } \\ & \text { Do they have risks or other limitations? }\end{aligned}$
treatment measures

considered actionable? Is it at the age at which the prevention or surveillance should be initiated?

Current recommendations on the age at which preventive measures and/or surveillance should be initiated are often based on the youngest age at which complications have been described in the literature, even if such cases are exceptional (Supplementary Table S2). For example, it is recommended that surveillance for complications of Von Hippel-Lindau syndrome should start at one year of age, even though complications of Von Hippel-Lindau do not usually manifest until much later. Furthermore, most surveillance and prevention recommendations are based on expert consensus, and are not evidence based. This lack of quality evidence is at least in part related to the difficulty of accruing evidence in the context of rare diseases.

In our analysis, we looked at the mode of inheritance, the percentage of de novo pathogenic variants, how obvious the clinical manifestations typically are, and the lifetime penetrance for each disorder. These characteristics influence the likelihood of benefit of the secondary finding. For example, unless the patient is adopted, for a number of autosomal dominant disorders, there may already be a known family history. In that situation, one could argue that it is not appropriate to use resources to screen for disorders that could be detected through family history. In contrast, patients with an autosomal recessive disorder often do not have a family history of the disorder. Autosomal recessive disorders are underrepresented in the ACMG list of reportable disorders, while a number of childhood-onset treatable genetic disorders are autosomal recessive disorders. Wilson's disease was added in the most recent update of the list. ${ }^{7}$ Ataxia with vitamin $\mathrm{E}$ deficiency is not on the ACMG list, but is another example of a childhood-onset autosomal recessive disorder for which adverse outcomes can be prevented if it is diagnosed presymptomatically. ${ }^{25}$

There is growing evidence that the identification of an secondary finding in an unselected individual with no family history of the disorder makes it difficult to determine the potential impact of that finding in that particular individual, particularly if the disorder has low penetrance and/or wide variability in expressivity. ${ }^{26,27}$ If that individual is an asymptomatic child, the prediction of future disease risk is on a longer time horizon and therefore even more challenging. The advent of large-scale sequencing has led to an expansion of clinical phenotypes for numerous disorders. ${ }^{28-30}$ This includes the discovery of individuals with a confirmed disease-causing pathogenic variant, but with a much milder phenotype than the one traditionally described or even absence of the documented phenotype. ${ }^{31}$ In addition, some pathogenic variants previously classified as disease causing are significantly more common in the general population than was previously thought, leading to reclassification of these pathogenic variants as variations of unclear clinical significance or benign. ${ }^{32,33}$ It is unclear how the identification of such a pathogenic variants in an asymptomatic child should be managed. Moreover, the phenotypes of disorders with a broad range of ages of onset, such as genetic aortopathies, arrhythmogenic disorders, cardiomyopathies, and cancer predisposition syndromes, are probably influenced by modifier genes. It is reasonable to question whether the prognosis for an asymptomatic child without a positive family history (hence on a genetic background more likely to be poor in deleterious genetic modifiers) is comparable to the expected prognosis in a child with a positive family history (hence on a genetic background more likely to be rich in deleterious genetic modifiers). If the risk of ever developing symptoms for the asymptomatic child with a negative family history is indeed lower, this should be balanced against the risks of a medical intervention and the risks of disclosure of an secondary finding. In such situations, we may be subjecting children to unnecessary and possibly risky surveillance or treatments without clear evidence of the benefit, and potentially causing more harm than good. Clearly, these issues are challenging when disclosing secondary findings to unselected individuals at any age, but even more so when disclosing such results to children and adolescents who may not benefit in the short term and are thereafter labeled with a genetic risk of disease.

The availability of an intervention (treatment or surveillance) is not sufficient to consider a disorder to be actionable: evidence of effectiveness of this intervention (i.e., the certainty and magnitude of its benefit on health outcomes) is also needed. ${ }^{34}$ Different thresholds for effectiveness can lead to different conclusions about which disorders are considered 
actionable. The highest threshold for effectiveness, as favored by the US Preventive Services Task Force and the Cochrane group, is supported by evidence from multiple clinical trials of a positive impact of surveillance or preventive measures on health outcomes. ${ }^{34}$ Because most diseases included in the ACMG panel are rare, clinical trials are difficult to conduct and therefore few genetic disorders meet this threshold. As seen in Table 3, most recommendations are based on verylow-to-low-quality evidence, and a few are based on evidence of moderate quality. A slightly less stringent threshold would be to consider disorders to be actionable if professional guidelines about treatment and surveillance are available, even if recommendations are based on observation studies or expert consensus. Some of the disorders in the ACMG panel meet this threshold, but not all do, and many available guidelines are not from professional societies, but rather by expert groups. Finally, the lowest threshold would be the availability of any surveillance or treatment proposed in the literature based on our current understanding of pathophysiology, whether or not there is evidence of its effectiveness. All disorders in the list would meet this threshold, but the lack of supporting evidence for proposed interventions may lead different clinicians to be more or less aggressive, and to make variable management decisions about an secondary finding in that situation.

In addition to health outcomes, other potential benefits and harms need to be weighed in the balance. Available empirical evidence has not shown persistent psychological harms of disclosure in children undergoing predictive testing for a positive family history of a given disorder. ${ }^{33}$ However, it remains to be seen whether disclosing a potential health risk to a child who did not expect it on the basis of family history is as benign in the long term. ${ }^{35}$ Anecdotally, clinicians report that most patients cope well with the disclosure of unanticipated results, but that a small subset of patients experience significant psychological and social harms from the disclosure of unexpected health risks. When balancing the risks and benefits, the potential psychological harm to a small minority of individuals associated with the disclosure of secondary findings should not be minimized, especially since the benefits of disclosing pathogenic variants in deleterious genes are unclear when we have no clear estimate of the penetrance and expressivity of such pathogenic variants in populations unselected for family history. Ethical issues about the restriction of future autonomy should also be considered. For children and adolescents, there needs to be more research on the potential health, psychological, and social impacts of the disclosure of secondary findings that are presumed to be pathogenic based on available data, but for which our ability to predict clinical outcomes is at best limited and at worse unclear.

Canada has a publicly funded health-care system with finite resources. We think there should be no obligation to search for disease-causing pathogenic variants in genes outside clinical indications for testing. Variant interpretation is a complex task that requires time and resources. Establishing a standard of mandatory reporting corresponds to performing opportunistic screening with all of its inherent obligations, including ensuring that individuals with abnormal results have access to appropriate evaluation and long-term management. The evaluation and follow-up of individuals found to carry pathogenic variants also requires access to specialist care and the use of health-care resources. The use of a mandatory reportable gene list imposes an additional burden on scarce resources, whereas only evaluating variants in genes that are stumbled on when looking for the patient's disorder minimizes this burden.

When deciding whether to include a disorder in a list of secondary findings that should be disclosed in children and adolescents, we believe the highest threshold of evidence of benefit for the child should be used. Most disorders included in the ACMG gene list do not meet population screening criteria, at least in childhood. We found that key elements in the evaluation process are the penetrance of the clinical manifestations amenable to treatment or prevention and the strength of the evidence supporting the efficacy of the intervention in mitigating the effects of these clinically significant manifestations. We propose that: (i) the proportion of cases with onset in childhood is a better indicator of the potential impact of actionability in childhood than the average age of onset; and (ii) disclosure in childhood should be limited to disorders for which a majority of cases present in childhood and for which interventions are supported by evidence of at least moderate quality. Among the ACMG list, the disorders that meet these criteria are multiple endocrine neoplasia type 2, retinoblastoma, tuberous sclerosis complex, Marfan syndrome, and Wilson's disease. Actively reporting secondary findings (i.e., performing opportunistic screening) for all other disorders should remain optional in childhood. Other diseases not currently on the ACMG list could also be considered, provided quality evidence supports actionability in childhood.

This proposal represents a cautious approach to the disclosure of secondary findings in childhood, with a focus on the clear benefits for the child. Others could argue that there may be benefits to disclosing secondary findings for disorders that only present in childhood in a minority of cases. Although a small proportion would benefit from early diagnosis, the benefits would be less clear for the group as a whole, since the majority of identified cases would not benefit from being identified in childhood, may actually be subjected to unnecessary interventions, and may also suffer from psychological or social harms of early identification. For lateonset disorders, there would not only need to be clear evidence of the benefit of intervention in childhood, but the threshold for the quality of the evidence should be held to a higher standard. Indeed, if we submit children to preventive measures years before the onset of disease, we need to have clear evidence that the intervention is effective. Lowering the threshold for the quality of the evidence of effectiveness for recommended interventions would add disorders to the list, but the clinical utility of disclosure for these other disorders in 
childhood would not be as clear. Indeed, Table 3 shows that the evidence of effectiveness is low for all of the disorders that occur in childhood in fewer than half of the cases. The evidence is at least of moderate quality for some of the disorders that are rare or exceptional in childhood, but either the available evidence is for adult-onset interventions and surveillance, or treatment in childhood is not recommended for most of these disorders.

Opportunistic screening in childhood leads to lifelong surveillance and treatment and should be supported by clear evidence of long-term benefits. The decision to offer optional opportunistic screening from a health-system perspective will depend, among other factors, on the availability of resources to perform such screening and ensure proper follow-up of identified cases in a context of scarce resources.

\section{ELECTRONIC SUPPLEMENTARY MATERIAL}

The online version of this article (https://doi.org/10.1038/s41436018-0034-4) contains supplementary material, which is available to authorized users.

\section{ACKNOWLEDGMENTS}

A.-M.L. received a Canadian Institutes of Health Research New Investigator Salary support grant and FRQS starting grant for new investigators (junior 1 level). Both authors were members of the Canadian College of Medical Geneticists' working group on secondary findings in genomic sequencing, which was active from 2013-2015 and led to the Canadian College of Medical Geneticists' position statement on the clinical application of genome-wide sequencing for monogenic diseases in Canada in 2015. The authors' work on this manuscript was initiated to answer questions raised during the discussions of this working group; therefore, the authors thank the other members of the working group for raising these questions. However, the work presented in this article has been performed independently from both the working group and the Canadian College of Medical Geneticists.

\section{DISCLOSURE}

The authors declare no conflicts of interest.

\section{REFERENCES}

1. Boycott KM, Rath A, Chong JX, et al. International cooperation to enable the diagnosis of all rare genetic diseases. Am J Hum Genet. 2017;100:695-705.

2. Amendola LM, Dorschner MO, Robertson PD, et al. Actionable exomic secondary findings in 6503 participants: challenges of variant classification. Genome Res. 2015;25:305-315.

3. Dorschner MO, Amendola LM, Turner EH, et al. Actionable, pathogenic secondary findings in 1,000 participants' exomes. Am J Hum Genet. 2013:93:631-640.

4. Green RC, Berg JS, Grody WW, et al. ACMG recommendations for reporting of secondary findings in clinical exome and genome sequencing. Genet Med. 2013;15:565-574.

5. Van El CG, Cornel MC, Borry $P$, et al. Whole-genome sequencing in health care: recommendations of the European Society of Human Genetics. Eur J Hum Genet. 2013;21:580-4.

6. Boycott K, Hartley T, Adam S, et al. The clinical application of genomewide sequencing for monogenic diseases in Canada: position statement of the Canadian College of Medical Geneticists. J Med Genet 2015;52:431-7

7. Kalia SS, Adelman K, Bale SJ, et al. Recommendations for reporting of secondary findings in clinical exome and genome sequencing, 2016 update (ACMG SF V2.0): a policy statement of the American College of Medical Genetics and Genomics. Genet Med. 2017;19:249-55.

8. Wolf SM, Annas GJ, Elias S. Point-counterpoint. Patient autonomy and secondary findings in clinical genomics. Science. 2013;340:1049-50.

9. Burke $\mathrm{W}$, Antommaria $\mathrm{AH}$, Bennett $\mathrm{R}$, et al. Recommendations for returning genomic secondary findings? We need to talk! Genet Med. 2013;15:854-9.

10. American College of Medical Genetics and Genomics. secondary findings in clinical genomics: a clarification. Genet Med. 2013;15:664-6.

11. Hehir-Kwa JY, Claustres M, Hastings RJ, et al. Towards a European consensus for reporting secondary findings during clinical NGS testing. Eur J Hum Genet. 2015;23:1601-6.

12. American Society of Human Genetics Board of Directors, American College of Medical Genetics Board of Directors. Points to consider: ethical, legal, and psychosocial implications of genetic testing in children and adolescents. Am J Hum Genet. 1995;57:1233-41.

13. Committee on Bioethics, Committee on Genetics, American College of Medical Genetics and Genomics Social, Ethical, and Legal Issues Committee. Ethical and policy issues in genetic testing and screening of children. Pediatrics. 2013;131:620-2.

14. Botkin JR, Belmont JW, Berg JS, et al. Points to consider: ethical, legal, and psychosocial implications of genetic testing in children and adolescents. Am J Hum Genet. 2015;97:6-21.

15. The Genetic Information Nondiscrimination Act of 2008. https://www. eeoc.gov/laws/statutes/gina.cfm. Accessed 30 August 2017.

16. Canada Health Act (R.S.C., 1985, c. C-6). http://laws-lois.justice.gc.ca/ eng/acts/c-6/FullText.html. Accessed 30 August 2017.

17. Genetic Non-Discrimination Act (S.C. 2017, c. 3). http://laws-lois.justice. gc.ca/eng/AnnualStatutes/2017_3/. Accessed August 30, 2017.

18. Wakefield CE, Hanlon LV, Tucker KM, et al. The psychological impact of genetic information on children: a systematic review. Genet Med. 2016;18:755-62.

19. Berg JS, Foreman AK, O'Daniel JM, et al. A semiquantitative metric for evaluating clinical actionability of secondary or secondary findings from genome-scale sequencing. Genet Med. 2016;18:467-75.

20. Moret C, Mauron A, Fokstuen S, et al. Defining categories of actionability for secondary findings in next-generation sequencing. J Med Ethics. 2017:43:346-9.

21. Wilson JMG, Jungner G. Principles and Practice of Screening for Disease. Geneva: WHO 1968.

22. Andermann A, Blancquaert I, Beauchamp S, et al. Revisiting Wilson and Jungner in the genomic age: a review of screening criteria over the past 40 years. Bull World Health Organ. 2008;86:317-9.

23. Descamps OS, Tenoutasse $S$, Stephenne $X$, et al. Management of familial hypercholesterolemia in children and young adults: consensus paper developed by a panel of lipidologists, cardiologists, paediatricians, nutritionists, gastroenterologists, general practitioners and a patient organization. Atherosclerosis. 2011;218:272-80.

24. American Society of Clinical Oncology. American Society of Clinical Oncology policy statement update: genetic testing for cancer susceptibility. J Clin Oncol. 2003;21:2397-406.

25. Ataxia with Vitamin E Deficiency. In: Pagon RA, Adam MP, Ardinger HH, et al. (eds). GeneReviews ${ }^{\circledR}$ [Internet]. University of Washington: Seattle, 1993.

26. Warren-Gash C, Kroese M, Burton $\mathrm{H}$, et al. Implications of using whole genome sequencing to test unselected populations for high risk breast cancer genes: a modelling study. Hered Cancer Clin Pract. 2016;14:12.

27. Van Driest SL, Wells QS, Stallings S, et al. Association of arrhythmiarelated genetic variants with phenotypes documented in electronic medical records. JAMA. 2016;315:47-57.

28. Tian $X$, Liang WC, Feng $Y$, et al. Expanding genotype/phenotype of neuromuscular diseases by comprehensive target capture/NGS. Neurol Genet. 2015;1:e14

29. Grandone A, Torella A, Santoro C, et al. Expanding the phenotype of RTTN variations: a new family with primary microcephaly, severe growth failure, brain malformations and dermatitis. Clin Genet. 2016;90:445-50.

30. Jaron $\mathrm{R}$, Rosenfeld N, Zahdeh $\mathrm{F}$, et al. Expanding the phenotype of CRB2 mutations-a new ciliopathy syndrome? Clin Genet. 2016;90: 540-44. 
31. Chen R, Shi L, Hakenberg J, et al. Analysis of 589,306 genomes identifies individuals resilient to severe Mendelian childhood diseases. Nat Biotechnol. 2016;34:531-38.

32. Andreasen C, Nielsen JB, Refsgaard L, et al. New population-based exome data are questioning the pathogenicity of previously cardiomyopathy-associated genetic variants. Eur J Hum Genet. 2013;21:918-28.

33. Refsgaard L, Holst AG, Sadjadieh G, et al. High prevalence of genetic variants previously associated with LQT syndrome in new exome data. Eur J Hum Genet. 2012;20:905-8.
34. Sawaya GF, Guirguis-Blake J, LeFevre M, et al. U.S. Preventive Services Task Force. Update on the methods of the U.S. Preventive Services Task Force: estimating certainty and magnitude of net benefit. Ann Intern Med. 2007;147:871-5.

35. Wynn J, Ottman R, Duong J, et al. Diagnostic exome sequencing in children: a survey of parental understanding, experience and psychological impact. Clin Genet. 2017;93:1039-48. 